\title{
Real-time 2.5Gbit/s spatial circuit switching on W-band wireless links
}

Rodríguez, Sebastián; Morales Vicente, Alvaro; Gallardo, Omar; Vegas Olmos, Juan José; Tafur Monroy, Idelfonso

Published in:

Optical Engineering

Link to article, DOI:

10.1117/1.OE.56.2.026104

Publication date:

2017

Document Version

Publisher's PDF, also known as Version of record

Link back to DTU Orbit

Citation (APA):

Rodríguez, S., Morales Vicente, A., Gallardo, O., Vegas Olmos, J. J., \& Tafur Monroy, I. (2017). Real-time 2.5 Gbit/s spatial circuit switching on W-band wireless links. Optical Engineering, 56(2), [026104].

https://doi.org/10.1117/1.OE.56.2.026104

\section{General rights}

Copyright and moral rights for the publications made accessible in the public portal are retained by the authors and/or other copyright owners and it is a condition of accessing publications that users recognise and abide by the legal requirements associated with these rights.

- Users may download and print one copy of any publication from the public portal for the purpose of private study or research.

- You may not further distribute the material or use it for any profit-making activity or commercial gain

- You may freely distribute the URL identifying the publication in the public portal 


\title{
Optical Engineering
}

\section{Real-time 2.5 Gbit/s spatial circuit switching on W-band wireless links}

\author{
Sebastián Rodríguez \\ Álvaro Morales \\ Omar Gallardo \\ Juan José Vegas Olmos \\ Idelfonso Tafur Monroy
}




\title{
Real-time $2.5 \mathrm{Gbit} / \mathrm{s}$ spatial circuit switching on W-band wireless links
}

\author{
Sebastián Rodríguez, ${ }^{a, *}$ Álvaro Morales, ${ }^{a}$ Omar Gallardo, ${ }^{a}$ Juan José Vegas Olmos, ${ }^{a}$ and Idelfonso Tafur Monroy \\ ${ }^{a}$ Technical University of Denmark, Department of Photonics Engineering, Kongens Lyngby, Denmark \\ ' ITMO University, Department of Photonics and Optical Information Technologies, St. Petersburg, Russia
}

\begin{abstract}
A spatial circuit switching system based on a beam steering application for W-band wireless links is proposed and experimentally demonstrated. The system enables two simultaneous transmissions of a $2.5 \mathrm{Gbit} / \mathrm{s}$ data signal over a carrier of $81 \mathrm{GHz}$, while allowing the receiver to dynamically switch between them. The performance of the system is tested with the real-time measurements of the BER, achieving values below the FEC limit for $7 \%$ of overhead and serving to prove the viability of wireless spatial circuit switching in the next generation of wireless access networks. @ 2017 Society of Photo-Optical Instrumentation Engineers (SPIE) [DOI: 10.1117/1.OE.56 .2.026104]
\end{abstract}

Keywords: switching; millimeter waves; optical communications.

Paper 161574 received Oct. 10, 2016; accepted for publication Jan. 17, 2017; published online Feb. 6, 2017.

\section{Introduction}

As we move toward the next generation of mobile networks, higher frequency bands have become more attractive due to higher bandwidth availability. The current focus of the $5 \mathrm{G}$ systems is to use the millimeter wave bands (30 to $300 \mathrm{GHz}$ ) for the wireless link between the network and the user equipment (UE). ${ }^{1}$ To generate and transport the millimeter wave signals to the radio units, radio-over-fiber (RoF) techniques have been proposed and studied to serve as the backbone technology in the back- and fronthaul networks. $^{2-4}$ The implementation of RoF has allowed the network to add extra capacity, control, and flexibility with for example optical switches and labels on $\mathrm{RoF}^{4-6}$ or multicore fibers. $^{7}$

Nevertheless, there are still some challenges in the wireless transmission of the signals in the W-band (75 to $110 \mathrm{GHz}$ ), one being the high attenuation at high frequencies. As an example, the attenuation for a distance of $2 \mathrm{~m}$ is $76.63 \mathrm{~dB}$. To compensate for the attenuation, the $\mathrm{W}$ band systems must use both high amplification stages and high gain antennas. ${ }^{3}$ Therefore, the selection of antennas for W-band systems is constrained to antennas with high directivity, which will impact the total coverage of the base transceiver stations (BTS). Up until now, the total coverage has been increased with cell sectorization, ${ }^{8}$ meaning that the total coverage area of the station is divided between two or more transceivers in the same BTS. However, the same implementation in W-band will require a large number of simultaneous transceivers in the BTS. In response, the use of beam steering techniques has been previously presented to enhance coverage and communication links in both uplink and downlink. ${ }^{1,8,9}$

In this paper, we propose and perform the real-time measurements of a circuit switching application for W-band (75 to $110 \mathrm{GHz}$ ) wireless links, by combining the generation of millimeter-wave wireless signals based on RoF techniques

*Address all correspondence to: Sebastián Rodríguez, E-mail: juse @ fotonik. dtu. $\mathrm{dk}$ with a beam steering application. This switching application allows the radio access unit (RAU) to dynamically select an area to transmit the wireless signal, increasing its total coverage adding flexibility and reliability to the next generation of radio access networks. The remainder of the paper is structured as follows: Sec. 2 describes the proposed system architecture. Section 3 describes the experimental setup and Sec. 4 discusses the experimental results. Finally, Sec. 5 summarizes the results and discusses the advantages of the proposed system.

\section{Spatial Switching by Antenna Steering}

Figure 1 presents the proposed scenario for the sectorization of a W-band radio access network. The network follows the standard centralized area network (C-RAN) architecture. In the C-RAN, the central office generates and processes the wireless signal and then transports it to different RAU that are dispersed through the network. The RAUs are used only as an interface between the optical fronthaul and the wireless link to connect with the UEs.

In each RAU, the total coverage is divided into various sectors and will have one transceiver creating the channel link. The antennas will illuminate only one position of the sector at a time due to the limitations on its beam width. However, each of the antennas will have implemented a beam steering application, which will allow a single antenna to spatially switch between different illuminated regions, covering the full sector.

\section{Experimental Setup}

Figure 2 shows the setup used in the proof of principle experiment. A $2.5 \mathrm{Gbit} / \mathrm{s} 2^{15}-1$ bit long pseudorandom bit sequence (PRBS15) nonreturn-to-zero (NRZ) signal from a pulse pattern generator (PPG) is used to modulate the optical carrier of a small form factor pluggable (SFP+) module. The modulated optical signal travels $10 \mathrm{~km}$ of standard singlemode fiber and then is combined with an external cavity

0091-3286/2017/\$25.00 @ 2017 SPIE 


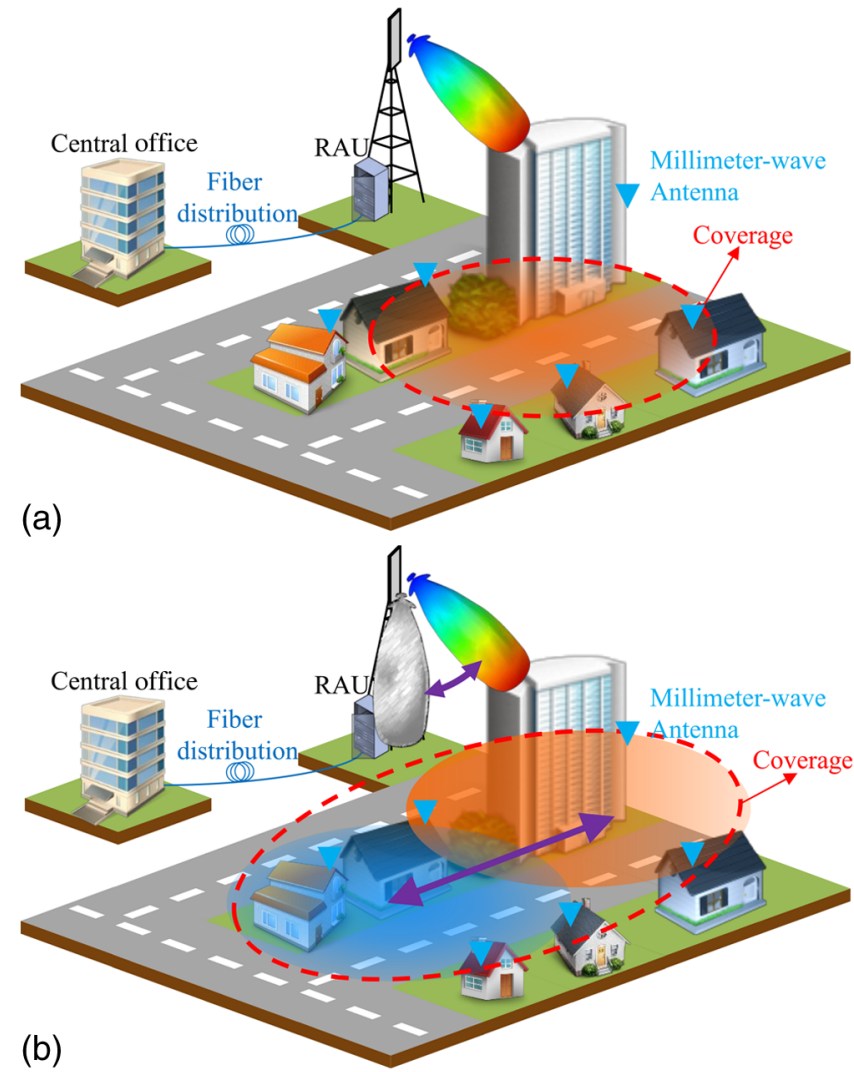

Fig. 1 Proposed scenario of RoF and wireless circuit switching. Steering of the main lobe of the antenna will enable the RAU to switch to a different region of the sector, increasing the total coverage by sectorizating the cell. (a) Normal coverage of the antena and (b) expanded coverage with circuit switching.

laser, which serves as a local oscillator (LO) with the optical input power adjusted with a variable optical attenuator. The two optical signals are boosted with an erbium-doped fiber amplifier (EDFA). A second VOA is placed for bit-error-rate (BER) measurements. Afterward, the signal is divided using a power splitter and delivered to two transmitters. The transmitters are composed of a high bandwidth photodiode (PD), a medium power amplifier (MPA) of gain $8 \mathrm{~dB}$ and a $24 \mathrm{dBi}$ horn antenna. The PD (Finisar XPDV4120R with $90 \mathrm{GHz}$ of bandwidth) is used to perform the upconversion process by direct heterodyning. ${ }^{2,3}$ The upconversion processes will create a double side band modulated signal in the electrical domain with a central carrier of $81 \mathrm{GHz}$, resulting in the transmission on the $\mathrm{W}$-band (75 to $110 \mathrm{GHz}$ ). The electrical signal in each transmitter is then fed to the horn antennas to be wirelessly transmitted. In order to decorrelate the transmitters, a waveguide is placed between the PD and the antenna in the first transmitter.

At the receiver, a 24- $\mathrm{dBi}$ horn antenna recovers the signal and delivers it to a 40-dB gain low noise amplifier, before a Schottky diode-based W-band envelope detector (ED) downconverts the data signal. Then, a clock recovery stage is used to provide both data and timing information to a bit-errorrate tester (BERT), allowing the real-time measurements of the BER.

The implemented beam steering solution is a mechanical steering technique based on Stewart platform. The Stewart platform is a robot with high accuracy $( \pm 0.06 \mu \mathrm{m})$, on a 6-axis ( $X Y Z$, pitch, roll, and yaw) actuator system arranged in parallel between two platforms. The receiver is mounted over the Stewart platform, giving it $6 \mathrm{deg}$ of freedom and allowing it to select between the two transmitters. The full system will then act as a wireless switch, with the insertion losses (IL) and crosstalk (XT) defined as a function of the radiation pattern of the antenna and the distance between transmitters:

$$
\begin{aligned}
& \mathrm{IL}_{\mathrm{TXi}-\mathrm{RX}}=G_{\mathrm{TXi}} G_{\mathrm{RX}}\left(\frac{\lambda}{4 \pi R_{\mathrm{TX} i-\mathrm{RX}}}\right)^{2}, \\
& \mathrm{XT}_{\mathrm{TXi}-\mathrm{TXj}}=G_{\mathrm{TXj}} G_{\mathrm{RX}}(\theta)\left(\frac{\lambda}{4 \pi R_{\mathrm{TXj}-\mathrm{RX}}}\right)^{2} .
\end{aligned}
$$

Here $G_{\mathrm{TXi}}$ and $G_{\mathrm{RX}}$ are the gain of the transmitter and receiver, respectively, $G_{\mathrm{RX}}(\theta)$ refers to the gain at the angle in sight of the other transmitter, $\lambda$ is the wavelength in the air, and $R$ is the distance.

To test the system two cases were implemented: case A, with both transmitters in the same height, as in Fig. 3(a); and case B with the transmitters at different heights, exemplified in Fig. 3(b). As shown in Fig. 3(c), the distance between the

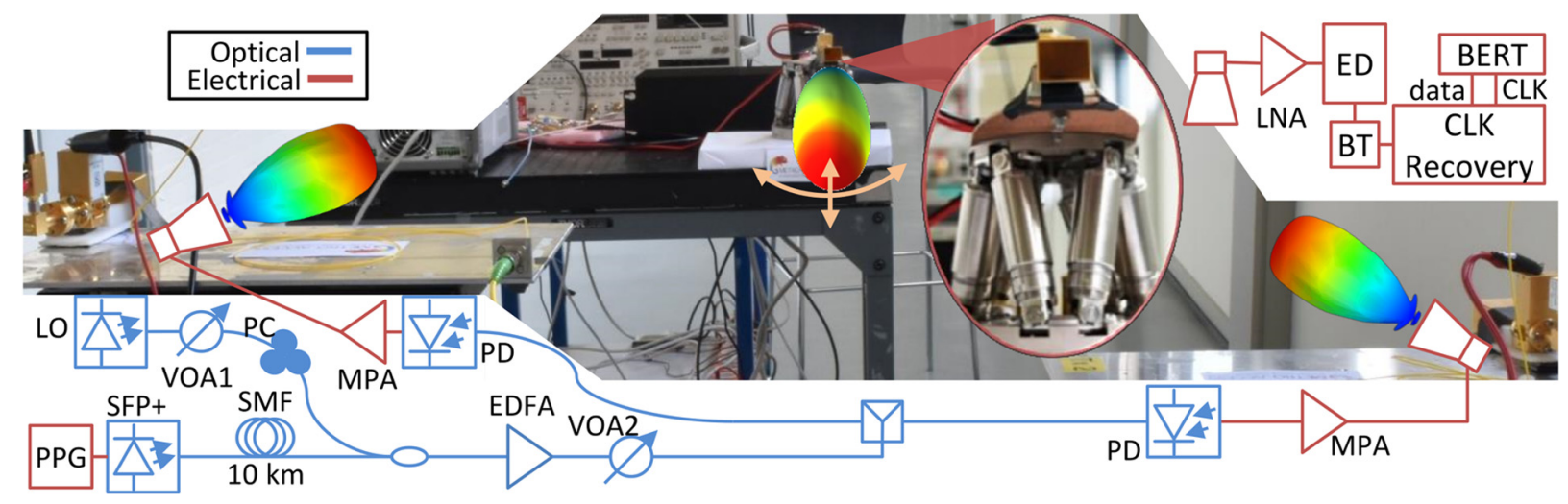

Fig. 2 Experimental setup. PPG, pulse pattern generator; SFP, small form factor pluggable; LO, local oscillator; VOA, variable optical attenuator; PC, polarization control; SMF, single mode fiber; EDFA, erbium-doped fiber amplifier; PD, photo diode; MPA, medium power amplifier; ED, envelope detector; BT, bias tee; CLK, clock; BERT, bit error rate tester. 
(a)

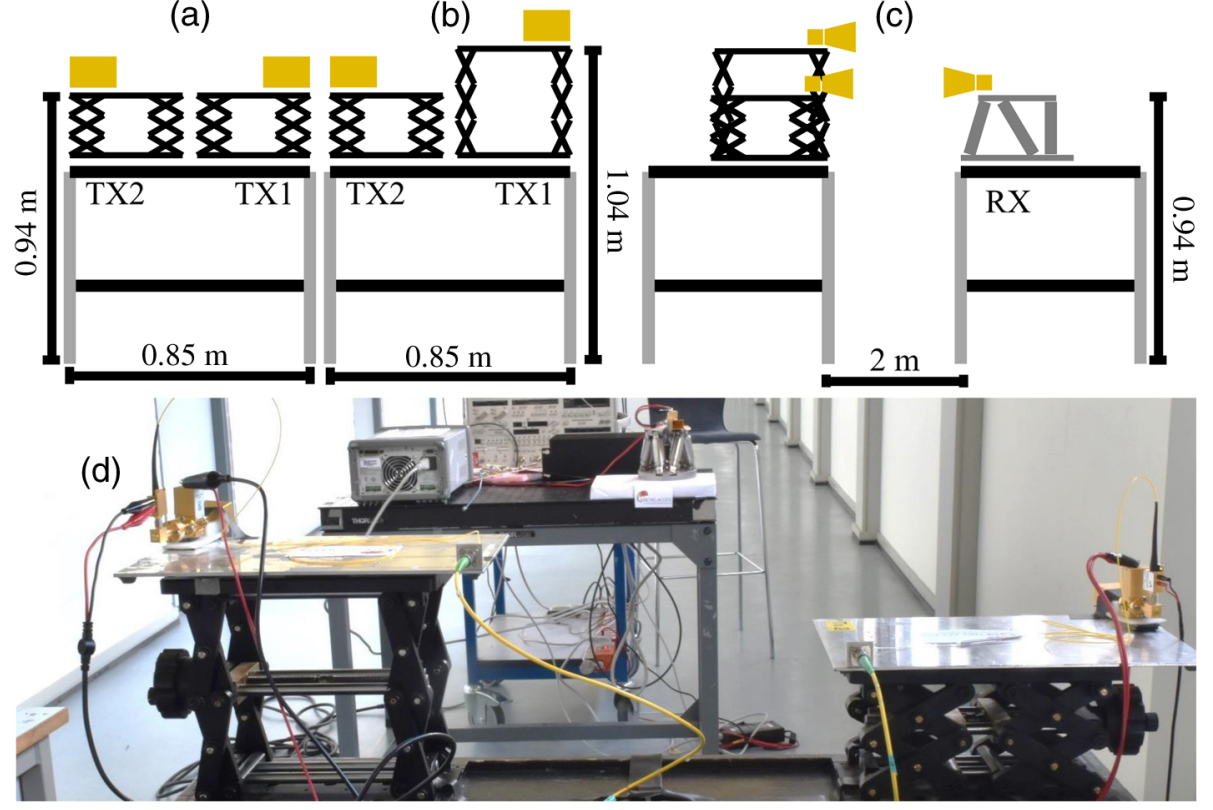

Fig. 3 Implementation of the experiment: (a) front view for case A, (b) front view for case B, (c) side view of the implementation, (d) real implementation. Video 1 shows the switching operation with the two antennas at the same height (MP4, $10 \mathrm{MB}$ ) [URL: http://dx.doi.org/10.1117/1.OE.56.2.026104.1].

receiver and the center of the two antennas was $2 \mathrm{~m}$. The final implementation for the second case can be seen in Fig. 3(d).

\section{Experimental Results}

In order to test the performance of the system, two sets of measurements were performed in each case. The first test was made to check the interference between the two channels. In this test, the receiver was aligned to each transmitter and the BER traces were measured in two steps: with both transmitters turned on and with only the one selected transmitting. The second test was performed to show the effects of the switching. Here the receiver was set to move between the transmitters and wait $10 \mathrm{~s}$ in each before moving again. During the experiment the transmitter 1 (TX1) received around $0.5 \mathrm{~dB}$ more optical power than the transmitter 2 (TRX2), stemming from differences in both the coupler and the fiber path before the PDs.

For the case A, the antennas were set at the same vertical distance. Video 1 shows the switching process. The measurements for the interference are shown in Fig. 4(a). For the case of the TRX1, having higher power, the effects of the TRX2 are not appreciable. Meanwhile, in the results for TRX2, a big aperture on the BER traces can be seen due to the presence of TRX1, lowering the performance of the system with a penalty of $0.4 \mathrm{~dB}$. The measures of the switching for this case are presented in Fig. 4(b). In both cases, the BER traces show similar tendencies as their static counterpart, but with a little extra attenuation of around $0.8 \mathrm{~dB}$, due to the small misalignment created while the antenna is moving.

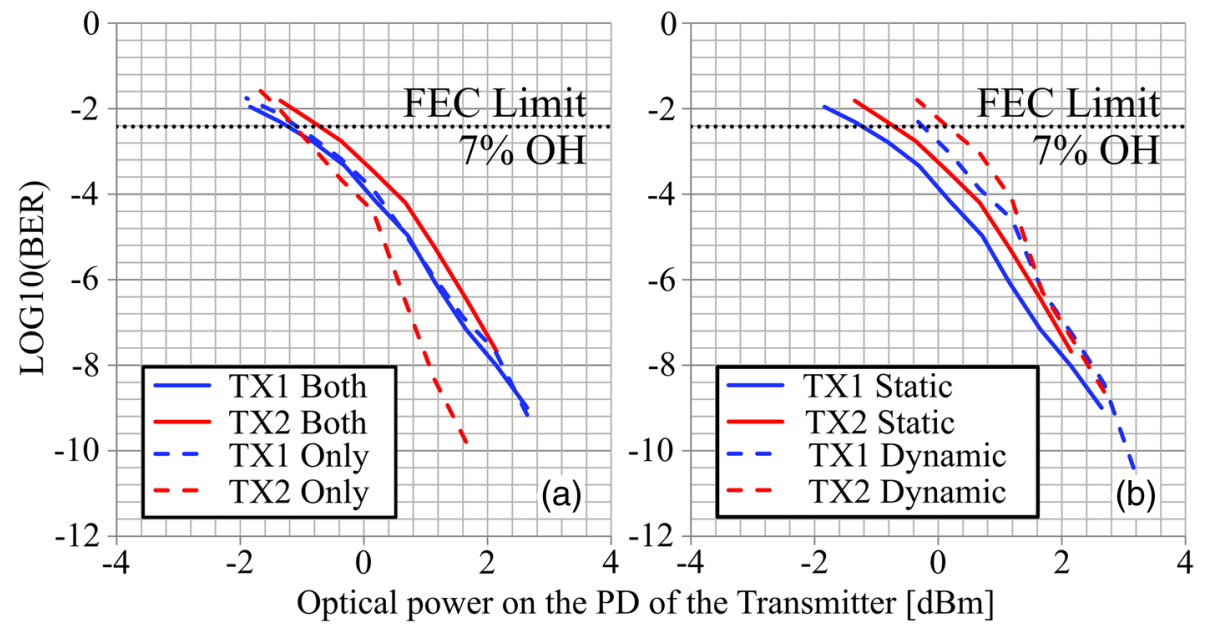

Fig. 4 Real time measurements of the BER versus the optical power on the PD of the transmitter, for the same height case: (a) Interference test and (b) static and dynamic switch mode comparison. Video 2 shows the switching operation with the two antennas with different height (MP4, 10.2 MB) [URL: http://dx.doi.org/10.1117/1.OE.56.2.026104.2]. 


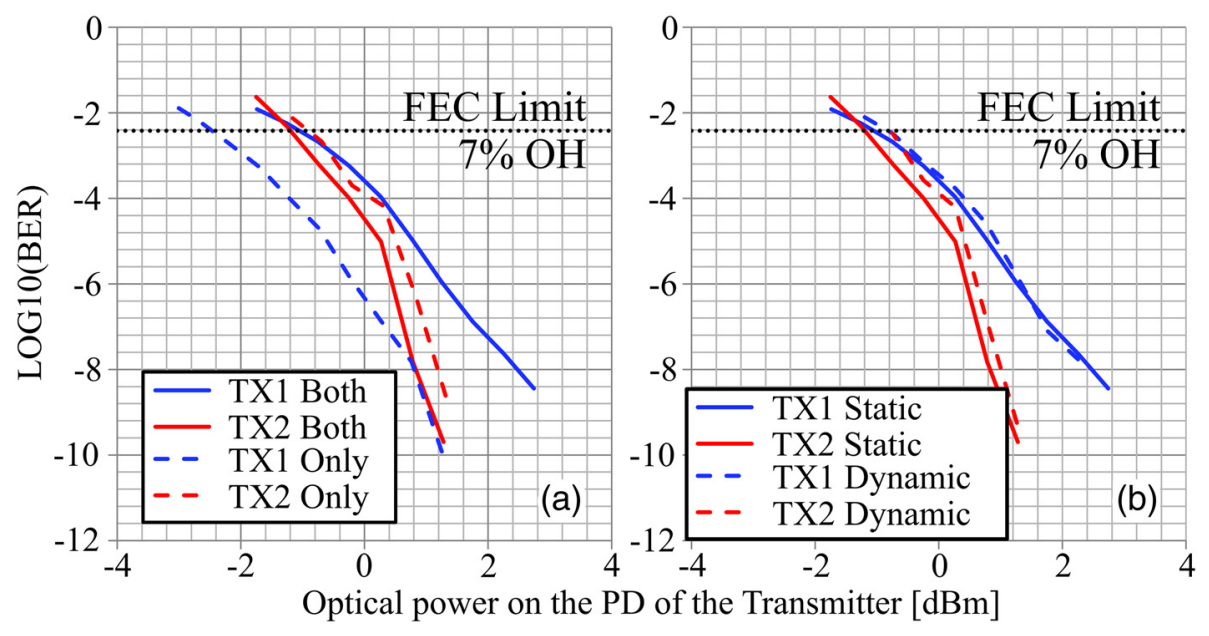

Fig. 5 Real-time measurements of the BER versus the optical power on the PD of the transmitter, for the different height case: (a) interference test and (b) static and dynamic switch mode comparison.

For case B, the height of TX1 was increased, effectively increasing the distance between the transmitters. Video 2 shows the switching operation of this case. Figure 5(a) shows the results for the interference test. For TX1, the presence of the second transmitter becomes more notable, causing a total penalty of $1.2 \mathrm{~dB}$. The TX2 is less affected, making only a difference of $0.2 \mathrm{~dB}$ between the two traces. Figure 5(b) shows the dynamic switching for this case. Once again the performance has a low penalty of $0.4 \mathrm{~dB}$ for the worst case and showing only as an extra attenuation on the system.

\section{Conclusions}

We have performed the real-time measurements of a $2.5 \mathrm{Gbit} / \mathrm{s}$ signal, wirelessly transmitted on the W-band (75 to $110 \mathrm{GHz}$ ), in two different cases of spatial circuit switching. The first one with the two transmitters at the same height and equally spaced from the receiver antenna, and the second increasing the height of one of the antennas. The spatial circuit switching was performed by moving the receiver antenna between two transmitters simultaneously enabled.

In both cases, the measured BER successfully achieved levels below the FEC limit for 7\% of overhead. These results demonstrate the viability of wireless spatial switching in the future generation of mobile networks. Although the test was done by moving the receiver, the theorem of reciprocity in antennas allows us to extend these results to the case of beam steering performed in the transmitter.

\section{Acknowledgments}

The authors would like to thank the Company Physik Instrumente (PI) for lending a Stewart platform to conduct the experiments. This work was partly founded by the Marie Skłodowska-Curie Innovative Training Network FiWiN5G supported by the European Union's Horizon 2020 research and innovation programme under grant agreement No. 642355 and the Innovative Training Network Celta project founded by the European Union's Horizon 2020 research and innovation programme under grant agreement No. 675683.

\section{References}

1. E. Dahlman et al., "5G wireless access: requirements and realization," IEEE Commun. Mag. 52(12), 42-47 (2014).

2. S. Rodriguez et al., "Photonic UP-convertion of carrierless amplitude phase signals for wireless communications on the Ka-band," Microwave Opt. Technol. Lett. 58(9), 2068-2070 (2016).

3. S. Rommel et al., "Outdoor W-band hybrid photonic wireless link based on an optical SFP+ module," IEEE Photonics Technol. Lett. 1 (2016).

4. J. J. V. Olmos and I. T. Monroy, "Reconfigurable radio-over-fiber networks [invited]," J. Opt. Commun. Networks 7(11), B23 (2015).

5. J. J. V Olmos et al., "Wireless and optical-integrated access network with peer-to-peer connection capability," IEEE Photonics Technol. Lett. 20(13), 1127-1129 (2008).

6. N. Chi et al., "Experimental characteristics of optical crosspoint switch matrix and its applications in optical packet switching," J. Lightwave Technol. 24(10), 3646-3653 (2006).

7. J. M. Galve et al., "Reconfigurable radio access networks using multicore fibers," IEEE J. Quantum Electron. 52(1), 1-7 (2016).

8. S. M. Razavizadeh, M. Ahn, and I. Lee, "Three-dimensional beamforming: a new enabling technology for 5G wireless networks," IEEE Signal Process. Mag. 31(6), 94-101 (2014).

9. A. Tatomirescu et al., "Beam-steering array for handheld devices targeting 5G," in Int. Symp. on Antennas and Propagation (ISAP 2015), pp. 1-4 (2015).

Sebastián Rodríguez received his BSc and MSc degrees in electronic engineering from the Pontificia Universidad Javeriana in 2011 and 2014, respectively. Currently, he is working as a Marie Curie Early Stage Researcher (ESR) at the Technical University of Denmark (DTU), under the FiWiN5G project. In his research, he works on the switching of $\mathrm{W}$-band signals for active and reconfigurable delivery. His interests include microwave photonics, optical switching, dynamic allocation, millimeter-wave communications, RF, and optical systems.

Álvaro Morales received his BSc degree in telecommunications technologies engineering from the University of Valladolid (UVa) in 2014, and his MSc degree in telecommunications engineering from UVa in 2016. He obtained a Marie Curie ESR scholarship in 2016 for doing his PhD studies at the DTU within CELTA project. In his research, he works on beam steering at $\mathrm{mm}$ waves and subterahertz frequencies.

Omar Gallardo received his BSc degree in electronics and automation engineering from the UVa in 2015. He obtained an Argo internship in 2015 in order to work as researcher for the Department of Photonics Engineering at the DTU. Currently he is studying for an MSc degree in electrical engineering with the Study Line in Automation and Robot Technology at the DTU.

Juan José Vegas Olmos received the PhD in 2006 from TU Eindhoven, Eindhoven, Netherlands. He holds BSc and MSc degrees 
in electronic engineering, a BA degree in economics, MA degree in arts, and an MBA. He was a JSPS fellow in Osaka University and worked as senior researcher at Hitachi Central Research Laboratory, Tokyo, Japan. In 2011, he joined the DTU, where since 2013 he has been an associate professor at the Department of Photonics Engineering.

Idelfonso Tafur Monroy graduated from the Bonch-Bruevitch Institute of Communications, St. Petersburg, Russia, in 1992, where he received his MSc degree in multichannel telecommunications. In 1996, he joined the electrical engineering department, Eindhoven University of Technology, Eindhoven, Netherlands, where he received the $\mathrm{PhD}$ in 1999 and worked as an assistant professor until 2006. He is currently a professor and head of the Metro-Access and Short Range Communications Group of the Department of Photonics Engineering, DTU. 